PREPARED FOR THE U.S. DEPARTMENT OF ENERGY, UNDER CONTRACT DE-AC02-76CH03073

PPPL-3757

PPPL-3757

UC-70

Enhanced Conversion of Thermal Electron Bernstein Waves

to the Extraordinary Electromagnetic Mode on the National Spherical Torus Experiment (NSTX)

by

G.Taylor, P.C. Efthimion, B. Jones, B.P. LeBlanc, J.R. Wilson, J.B. Wilgen, G.L. Bell, T.S. Bigelow, R. Maingi, D.A. Rasmussen, R.W. Harvey, A.P. Smirnov, F. Paoletti, and S.A. Sabbagh

October 2002
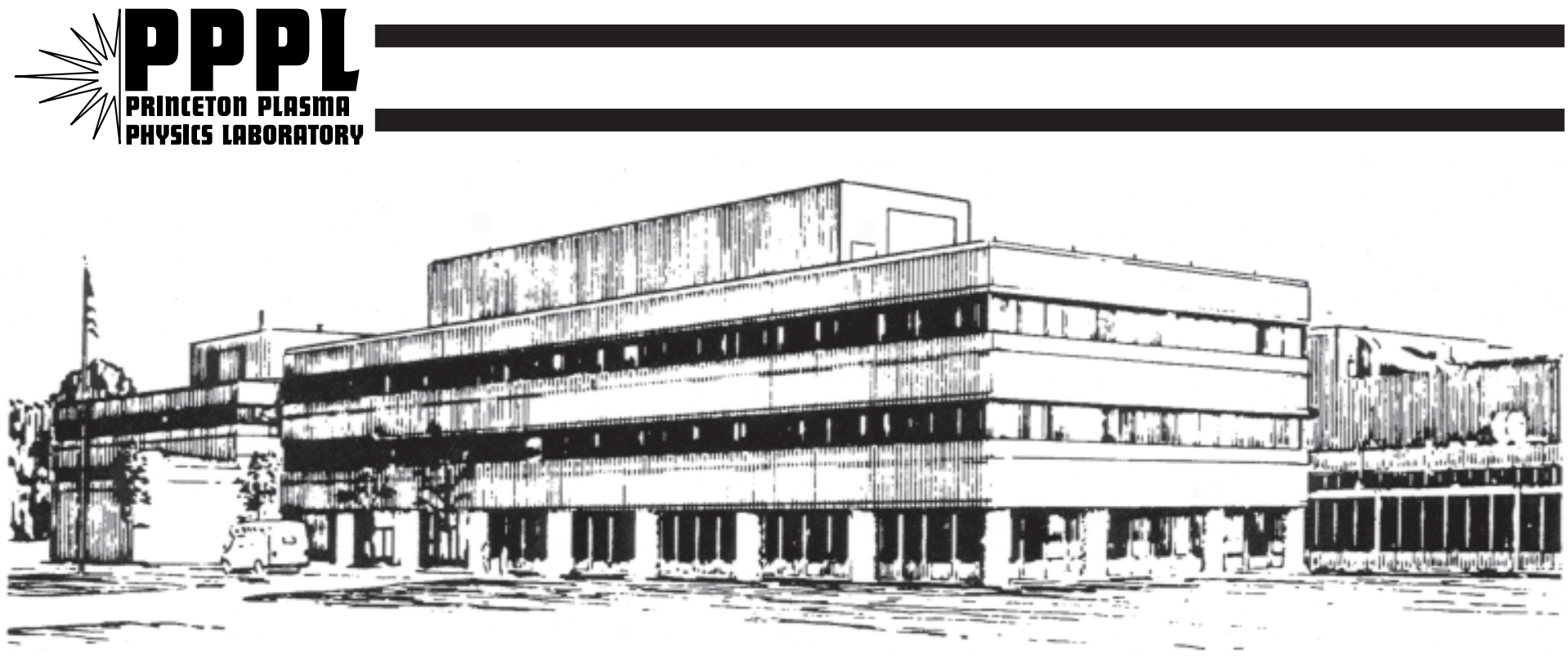

PRINCETON PLASMA PHYSICS LABORATORY PRINCETON UNIVERSITY, PRINCETON, NEW JERSEY 


\section{PPPL Reports Disclaimer}

This report was prepared as an account of work sponsored by an agency of the United States Government. Neither the United States Government nor any agency thereof, nor any of their employees, makes any warranty, express or implied, or assumes any legal liability or responsibility for the accuracy, completeness, or usefulness of any information, apparatus, product, or process disclosed, or represents that its use would not infringe privately owned rights. Reference herein to any specific commercial product, process, or service by trade name, trademark, manufacturer, or otherwise, does not necessarily constitute or imply its endorsement, recommendation, or favoring by the United States Government or any agency thereof. The views and opinions of authors expressed herein do not necessarily state or reflect those of the United States Government or any agency thereof.

\section{Availability}

This report is posted on the U.S. Department of Energy's Princeton Plasma Physics Laboratory Publications and Reports web site in Fiscal Year 2003. The home page for PPPL Reports and Publications is: http://www.pppl.gov/pub_report/

DOE and DOE Contractors can obtain copies of this report from:

U.S. Department of Energy

Office of Scientific and Technical Information

DOE Technical Information Services (DTIS)

P.O. Box 62

Oak Ridge, TN 37831

Telephone: (865) 576-8401

Fax: (865) 576-5728

Email: reports@adonis.osti.gov

This report is available to the general public from:

National Technical Information Service

U.S. Department of Commerce

5285 Port Royal Road

Springfield, VA 22161

Telephone: $1-800-553-6847$ or

(703) $605-6000$

Fax: (703) 321-8547

Internet: http://www.ntis.gov/ordering.htm 


\title{
Enhanced conversion of thermal electron Bernstein waves to the extraordinary electromagnetic mode on the National Spherical Torus Experiment (NSTX)
}

\author{
G. Taylor, P.C. Efthimion, B. Jones, B.P. LeBlanc, J.R. Wilson \\ Princeton Plasma Physics Laboratory, Princeton, New Jersey 08543, USA \\ J.B. Wilgen, G.L. Bell, T.S. Bigelow, R. Maingi, D.A. Rasmussen \\ Oak Ridge National Laboratory, Oak Ridge, Tennessee 08731, USA \\ R.W. Harvey, \\ CompX, Del Mar, CA 920314, USA \\ A.P. Smirnov \\ Moscow State University, Moscow, Russia \\ F. Paoletti, S.A. Sabbagh \\ Columbia University, New York, NY 10027, USA
}

\begin{abstract}
A four-fold increase in the conversion of thermal electron Bernstein waves (EBW) to the extraordinary mode (X-mode) was measured when the density scale length $\left(L_{n}\right)$ was progressively shortened by a local Boron nitride limiter in the scrape-off of an Ohmicallyheated National Spherical Torus Experiment (NSTX) plasma [M. Ono, S. Kaye, M. Peng, et al., Proceedings $17^{\text {th }}$ IAEA Fusion Energy Conference (IAEA, Vienna, Austria, 1999), Vol.3, p. 1135]. The maximum conversion efficiency approached $50 \%$ when $L_{n}$ was reduced to $0.7 \mathrm{~cm}$, in agreement with theoretical predictions that used locally measured $L_{n}$. Calculations indicate that it is possible to establish $L_{n}<0.3 \mathrm{~cm}$ with a local limiter, a value predicted to attain $\sim 100 \% \mathrm{EBW}$ conversion to the X-mode in support of proposed EBW heating and current drive scenarios.
\end{abstract}

PACS\# 52.55.Fa, 52.35.Hr 


\section{INTRODUCTION}

The National Spherical Torus Experiment (NSTX) [1] is a magnetically confined plasma device that operates at high electron densities $\left(1-5 \times 10^{19} \mathrm{~m}^{-3}\right)$ relative to its confining magnetic field $(<0.6 \mathrm{~T})$. In such a spherical torus $(\mathrm{ST})$ the electron plasma frequency far exceeds the electron cyclotron frequency and, as a consequence, electromagnetic waves at fundamental and low harmonic electron cyclotron frequencies cannot propagate within the plasma. Therefore conventional electron cyclotron emission (ECE) electron temperature profile $\left(T_{e}(R)\right)$ diagnostics, electron cyclotron heating $(\mathrm{ECH})$ and electron cyclotron current drive (ECCD) techniques cannot be used in an ST. In contrast, electron Bernstein waves (EBW) readily propagate in ST plasmas and are absorbed strongly at the ECE resonances [2]. These characteristics make EBWs potentially attractive as a $T_{e}(R)$ diagnostic or for $\mathrm{ECH}$ and $\mathrm{ECCD}$, but in order to propagate beyond the upper hybrid resonance (UHR), that surrounds the ST plasma, the EBWs must convert via one of two processes to either O-mode [3] or X-mode electromagnetic waves [4]. Efficient conversion to O-mode (B-X-O conversion) requires the superposition of the O-mode cutoff and the left-hand cutoff, which is accomplished by viewing the plasma obliquely at an optimum angle. On an ST this optimum angle varies dynamically due to large variations in the topology of the magnetic field during a discharge. On the other hand, the conversion of the EBW to the X-mode, the process studied here on NSTX and elsewhere [5, 6], involves viewing the plasma normal to the magnetic field and depends on the conversion of EBWs to the fast X-mode via the slow X-mode (B-X conversion). A cutoff-resonance-cutoff triplet, formed by the left hand cutoff of the slow X-mode, the UHR, and the right-hand cutoff of the fast X-mode, can allow the slow X-mode to tunnel through the UHR to the fast X-mode branch. The B-X conversion efficiency for $\mathrm{k}_{/ /}=0$ is given by [4]: 


$$
C=C_{\max } \cos ^{2}(\phi / 2+\theta)
$$

Here $\cos ^{2}(\phi / 2+\theta)$ is a phase factor relating to the phasing of the waves in the mode conversion region and $C_{\max }$ is the maximum mode conversion efficiency given by:

$$
C_{\max }=4 e^{-\pi \eta}\left(1-e^{-\pi \eta}\right)
$$

Here $\eta$ is a tunneling parameter. For magnetic scale lengths much greater than the density scale length at the UHR, as is typically the case near the last closed flux surface (LCFS) of ST plasmas:

$$
\eta \approx\left[\omega_{c e} L_{n} / c \alpha\right]\left(\sqrt{1+\alpha^{2}}-1\right)^{1 / 2}
$$

$$
\text { Here: } \quad \alpha=\left[\omega_{p e} / \omega_{c e}\right]_{U H R} \text { and } L_{n}=\left|\frac{n_{e}}{\mid d n_{e} / d R}\right|_{U H R}
$$

Here $\omega_{c e}$ and $\omega_{p e}$ are the electron cyclotron frequency and electron plasma frequency, respectively, $n_{e}$ is the electron density and $c$ is the velocity of light. It is apparent from equations (1) - (3) above that $C$ is sensitively dependent on $L_{n}$ at the UHR layer where the B-X mode conversion and tunneling occurs.

On NSTX, and other ST devices, fundamental EBW emission frequencies are typically resonant with a B-X conversion layer that lies in the plasma scrape off or just outside the LCFS and second harmonic EBW emission frequencies convert at or just outside the LCFS. Using the above equations, $\sim 100 \%$ mode conversion efficiency for fundamental EBW on NSTX is expected for $L_{n} \sim 0.2-0.8 \mathrm{~cm}$, depending on the confining magnetic field. However, in NSTX L-mode discharges $L_{n}$ is typically $2-5 \mathrm{~cm}$ just outside the LCFS, resulting in predicted $C<1 \%$. Even when there is a natural steepening of the edge density gradient during an H-mode, $L_{n}$ is still no shorter than $1.5 \mathrm{~cm}$ just outside the LCFS, resulting in a predicted $C \sim 10 \%$. It should be noted that earlier measurements of EBW 
mode conversion efficiency on NSTX during the H-mode phase are in relatively good agreement with this prediction [7].

Recent experiments on CDX-U, a small ST device at Princeton, have successfully shown that a radially adjustable local limiter surrounding the EBW antenna can produce $L_{n}$ values that are optimized for $\sim 100 \% \mathrm{~B}-\mathrm{X}$ conversion [8]. The local limiter is positioned outside the LCFS, so it is not the primary limiter for the plasma. This result has important implications, not only for an $\mathrm{EBW} T_{e}(R)$ diagnostic, but, as a result of the symmetry of the mode conversion process [9], also for EBW heating and current drive scenarios being considered for larger ST devices. Indeed, the demonstration of a resilient technique that maintains $\mathrm{B}-\mathrm{X}$ conversion efficiencies $\geq 80 \%$ is a necessary prerequisite for implementing a high power EBW heating or current drive system based on B-X conversion on a large ST machine.

In order to demonstrate that the technique developed on $\mathrm{CDX}-\mathrm{U}$ has potential for a larger ST device, an experiment was conducted on NSTX to shorten $L_{n}$ in the scrape off by progressively pushing the plasma out towards an array of Boron nitride limiter tiles which are integrated into the antenna used for high harmonic fast wave (HHFW) heating [10]. As the distance between the LCFS and the Boron nitride limiters was decreased, we observed a significant four-fold increase in B-X conversion efficiency for EBW emission measured with a microwave antenna situated between two of the HHFW antenna straps. During this experiment, an X-mode reflectometer near the location of the EBW antenna, provided time resolved measurements of $L_{n}$. This $L_{n}$ measurement, together with $T_{e}(R)$ measured by multi-point Thomson scattering, allowed a comparison of the measured $C$ to the predictions of B-X mode conversion theory [4].

Section II of this paper describes the experimental setup, section III presents examples of the measurements made during the experiment, section IV presents an analysis of these data and a comparison to theory and section $\mathrm{V}$ is a discussion of the results and future work. 


\section{EXPERIMENTAL SETUP}

The NSTX HHFW antenna extends poloidally about 50 degrees above and below the midplane and toroidally about a quarter of the way around the outboard side of the vacuum vessel [10]. The antenna has a total of 12 straps. The four straps surrounding the EBW antenna used for the measurements reported in this paper are shown in Fig. 1. Each strap is covered by a Molybdenum Faraday shield and separated from adjacent straps by Boron nitride protective tiles. Three circular microwave guides, shown in the close-up photograph in Fig.1, were installed between two of the antenna straps.

Figure 2 is a schematic diagram showing a side view of the three circular microwave guides. The central waveguide is on the horizontal midplane. The other waveguides are displaced above and below the midplane and tilted at 6 degrees to the horizontal. The upper two waveguides are used by a $6-27 \mathrm{GHz} \mathrm{X}$-mode reflectometer that measures the electron density in the range $1 \times 10^{11} \mathrm{~cm}^{-3}$ to $6 \times 10^{12} \mathrm{~cm}^{-3}$ every $100 \mu \mathrm{s}$ [11]. The lower waveguide was used for fundamental X-mode EBW radiometry. Also shown in this figure is the outer gap between the last closed flux surface and the boron nitride tiles which was adjusted by moving the plasma to vary $L_{n}$. The Boron nitride tiles extend 2-3 $\mathrm{mm}$ beyond the Molybdenum Faraday shields and effectively provide a local limiting surface in the vicinity of the EBW antenna when the plasma outer gap is $\leq 1 \mathrm{~cm}$. There is a $14 \mathrm{~cm}$ vertical break in the Boron nitride tiles to accommodate the three circular microwave guides.

A series of five Ohmically-heated, Deuterium plasmas with axial vacuum toroidal field, $B_{o}=0.4 \mathrm{~T}$ and plasma current, $I_{p}=800 \mathrm{kA}$ were programmed to have progressively smaller separations between the LCFS and the Boron nitride tiles surrounding the HHFW antenna. This outer gap separation was calculated using the EFIT [12, 13] magnetic equilibrium code. During the sequence of discharges the outer gap during the $I_{p}$ flattop was reduced from about $6 \mathrm{~cm}$ to less than $1 \mathrm{~cm}$ with otherwise similar plasma parameters. 
Figure 3 shows a comparison of the evolution of $I_{p}$ and outer gap separation for the two plasmas with the smallest and largest outer gap, shots 107979 (thick line) and 107975 (thin line), respectively. The shaded region in this figure, between 0.28 and $0.32 \mathrm{~s}$, indicates the time window over which the discharges were analyzed.

Figure 4 overlays electron temperature and density profiles measured by multi-point Thomson scattering [14] within the analysis time window, $0.28-0.32 \mathrm{~s}$, for the two shots shown in Fig 3. The plotted profiles are each the result of averaging a spline fit to two, 20point radial profiles measured at 0.293 and $0.310 \mathrm{~s}$. While there are some differences in the profiles, their shapes are similar. The major difference being the 5-6 cm outboard shift in shot 107979 relative to shot 107975 , which is consistent with the difference in the outer gap calculated by EFIT. For the five plasmas used in the forgoing analysis, central electron temperatures varied between $530 \mathrm{eV}$ and $575 \mathrm{eV}$ and central electron densities varied between $4.2 \times 10^{13} \mathrm{~cm}^{-3}$ and $4.8 \times 10^{13} \mathrm{~cm}^{-3}$, within the analysis time window. The errors on the $T_{e}$ and $n_{e}$ Thomson scattering measurements are $\pm 4 \%$.

EBW emission was acquired with an 8-12 GHz, frequency-swept, heterodyne radiometer [15] with an effective frequency resolution of $400 \mathrm{MHz}$. For the plasma parameters studied here, this frequency resolution corresponds to a major radial resolution of about $5 \mathrm{~cm}$ near the plasma axis. The EBW emission spectrum was measured every 100 $\mu$ s. EBW radiation temperatures $\left(T_{r a d}\right)$ quoted in this paper were derived from an absolute calibration of the radiometer with a chopped $77 \mathrm{~K}$ blackbody source. The absolute calibration setup included a mockup of the antenna, front-end waveguide, vacuum window and low-loss dielectric microwave cable used for the EBW emission measurements. The errors on the EBW $T_{\text {rad }}$ measurements are $\pm 15 \%$. 


\section{EBW MODE CONVERSION MEASUREMENTS}

Figure 5 shows the evolution of the central $T_{e}$ and EBW $T_{r a d}$ at $11.6 \mathrm{GHz}$ for shots 107975 and $107979.11 .6 \mathrm{GHz}$ is a frequency that, we will show later, corresponds to fundamental EBW emission from the magnetic axis. Between $0.2 \mathrm{~s}$ and $0.35 \mathrm{~s}$, central $T_{e}$, as measured by Thomson scattering every $17 \mathrm{~ms}$, increases from $400 \mathrm{eV}$ to about $550 \mathrm{eV}$. On shot 107975, the shot with an outer gap of about $6 \mathrm{~cm}$, the EBW $T_{r a d}$ at $11.6 \mathrm{GHz}$ (thin line) fluctuates around a value of about $50 \mathrm{eV}$. On shot 107979, the shot with an outer gap of less than $1 \mathrm{~cm}$, the EBW $T_{r a d}$ at $11.6 \mathrm{GHz}$ (thick line) fluctuates around a value of about $200 \mathrm{eV}$, even though the central $T_{e}$ evolution is similar to shot 107975 .

For the five shots studied in this experiment, $11.6 \mathrm{GHz}$ EBW emission converts to fast $\mathrm{X}$-mode at a density of $1 \times 10^{12} \mathrm{~cm}^{-3}$. Fig. 6(a) shows a plot of $L_{n}$ measured at a density of $1 \times 10^{12} \mathrm{~cm}^{-3}$ by the X-mode reflectometer for shots 107975 and 107979 between 0.28 and 0.32 s. $L_{n}$ exhibits large fluctuations on both shots. On shot $107975 L_{n}=1.95 \pm 0.45 \mathrm{~cm}$ whereas on $107979 L_{n}=0.70 \pm 0.25 \mathrm{~cm}$, consistent with the shorter scrape-off length expected for the shot with the smaller outer gap. The average EBW conversion efficiency was calculated for the time period 0.28 to $0.32 \mathrm{~s}$, a time period when plasma parameters remained relatively constant. Fig. 6(b) shows the experimentally inferred B-X conversion efficiency, $T_{r a d} / T_{e}$, at $11.6 \mathrm{GHz}$ plotted between 0.28 and $0.32 \mathrm{~s}$, for the same shots as Fig. 6(a). This assumes that the $11.6 \mathrm{GHz}$ emission measured by the EBW radiometer is emitted from a region near the magnetic axis and that the signal is entirely due to B-X mode conversion and tunneling. On shot $107975, T_{\text {rad }} / T_{e}=10 \pm 5 \%$ and on shot 107975 average $T_{\text {rad }} / T_{e}$ increased approximately four fold to $38 \pm 13 \%$. 


\section{ANALYSIS AND COMPARISON TO THEORY}

To determine if the $11.6 \mathrm{GHz} \mathrm{EBW}$ emission has its source near the magnetic axis, an EBW ray-tracing calculations was performed with the GENRAY code [16]. An EFIT [13] magnetic equilibrium from 107975 at $0.301 \mathrm{~s}$ was used for the calculation. The ray tracing calculation used the $n_{e}$ and $T_{e}$ profiles measured by Thomson scattering from 107975 , shown in Fig. 4. For the ray tracing calculations, a bundle of 12 rays were launched from 6 degrees below the midplane. Launch location for each ray was spread over a range of $n_{/ /}$ and poloidal angle to approximate the near-field pattern of the open-ended waveguide antenna used for the experiment. Figure 7 shows results from the ray-tracing calculation. Figure 7(a) shows the EBW ray paths projected onto a toroidal cross-section through the midplane and Fig. 7(b) shows the same EBW ray paths projected on to the poloidal crosssection. Rays are plotted until $99 \%$ of the EBW power is absorbed. The shaded region in Fig. 7(b) indicates the region where $>99 \%$ of the EBW power is deposited. This region has a major radial width of about $10 \mathrm{~cm}$, about twice the major radial resolution due to the finite bandwidth of the EBW radiometer. Since, as shown in Fig. 4(a), the center of the $T_{e}$ profile is relatively flat over a region extending about $40 \mathrm{~cm}$ in major radius about the magnetic axis, it is reasonable to use the core $T_{e}$ when calculating the inferred $\mathrm{B}-\mathrm{X}$ conversion efficiency, $T_{r a d} / T_{e}$, at $11.6 \mathrm{GHz}$.

The theoretical B-X conversion efficiency, $C$, was calculated from equations (1) - (3) for $11.6 \mathrm{GHz} \mathrm{EBW}$ emission and the parameters of the discharges employed for the experiment. Figure 8 shows a plot of both $C$ and $C_{\max }$ versus $L_{n}$, where the $L_{n}$ values here are averaged over the analysis window for each of the five shots in the outer gap scan. The maximum value of $C$ reaches $98 \%$ when $L_{n}$ falls to $2.5-3 \mathrm{~mm}$. The inferred B-X conversion efficiencies derived from the EBW $T_{r a d} / T_{e}$ measured in the experiment are also plotted in Fig. 8 (filled circles). $L_{n}$ attained in this experiment was about a factor of two longer than is needed for $\sim 100 \% \mathrm{~B}-\mathrm{X}$ conversion. Note that the error bars in Fig. 8 
actually represent the standard deviation of the fluctuations in $T_{r a d} / T_{e}$ and $L_{n}$ within the analysis time window, not the uncertainty in these quantities. The average conversion efficiency, $\left\langle C(t)>\right.$, and average maximum conversion efficiency, $\left\langle C_{\max }(t)>\right.$, within the analysis time window, were also calculated by using the measured $L_{n}(t)$ in equations (1) and (2), respectively. In Table $1<C(t)>$ and $\left\langle C_{\max }(t)>\right.$ and the standard deviation of the fluctuation in these quantities within the analysis time window are compared to $\left\langle T_{\mathrm{rad}} / T_{e}\right\rangle$ and standard deviation of its fluctuation. In general, while there are large fluctuations in all three quantities, the observed fluctuation in $T_{r a d} / T_{e}$ was smaller than would be expected given the measured fluctuation in $L_{n}$.

\section{DISCUSSION AND FUTURE WORK}

While the measured EBW mode conversion efficiencies from the experiment are in reasonable agreement with theory, there are some notable differences that need to be discussed. First, the measured efficiencies agree better with the theoretical $C_{\max }$ than with $C$ near $L_{n} \sim 1 \mathrm{~cm}$ (shot 107976 and 107977 in Table I). Second, the measured conversion efficiency is about a factor of three greater than $C$ or $C_{\max }$ for $L_{n} \sim 2 \mathrm{~cm}$ (shot 107975 in Table I). Third, we would expect the fluctuations in $T_{r a d} / T_{e}$ to correlate with fluctuations in $L_{n}$ at the B-X mode conversion layer, so that $T_{r a d} / T_{e}$ is a maximum when $L_{n}$ is a minimum. In fact, no such correlation is seen. It should be noted that in recent experiments on CDX$\mathrm{U}$ [17], a clear correlation between fluctuations in EBW $T_{\text {rad }}$ and $L_{n}$ was observed.

The behavior of the phase factor, $\cos ^{2}(\phi / 2+\theta)$, in equation 1 can be explained as follows; as $L_{n}$ changes, so does the spacing between the left- and right-hand cutoffs. These cutoffs bound the mode conversion region, acting as mirrors to form a cavity surrounding the UHR. Essentially, the X-mode forms standing waves, and if the spacing of the cutoff layers provides a multiple $\pi / 2$ phase shift across the cavity, the phase factor can

approach zero. As $L_{n}$ changes, there is an oscillation between constructive and destructive 
interference in the cavity. However, an implicit assumption of the B-X conversion model of ref. 4 is that $n_{/ /}=0$, but due to the finite angular acceptance pattern of the EBW antenna used in the experiment on NSTX, there will be rays that couple to the antenna with $n_{/ /} \neq 0$. Oblique propagation changes the phase factor for a given $L_{n}$ as the effective spacing of the cutoffs changes, therefore the emission received by the EBW antenna will be the result of the superposition of waves that experience different phase shifts in the mode conversion region. The functional dependence of $C$ on $L_{n}$ for these waves will be bounded by $C_{\max }$, which may explain why the observed dependence of $T_{r a d} / T_{e}$ on $L_{n}$ is in better agreement with the $L_{n}$ dependence of $C_{\text {max }}$, rather than $C$.

The discrepancy between the measured $T_{r a d} / T_{e}$ and the theoretically predicted B-X conversion for $L_{n} \sim 2 \mathrm{~cm}$ maybe the result of some emission from B-X-O conversion at oblique angles entering the EBW antenna via reflection from the vacuum vessel wall and right-hand cutoff. This has been noted before for EBW emission measurements on NSTX [7] and CDX-U [15]. If this is the case, some of the EBW emission measured at shorter $L_{n}$ may also result from B-X-O conversion, reducing the effective measured B-X conversion efficiency.

The lack of correlation between the fluctuation in $T_{r a d} / T_{e}$ and $L_{n}$ measured by the Xmode reflectometer suggests that while the reflectometer may be measuring $L_{n}$ in the vicinity of the EBW antenna it is not measuring $L_{n}$ fluctuations that are correlated with fluctuations in front of the antenna. Since the EBW mode conversion is occurring just a few centimeters in front of the reflectometer and EBW radiometer antennas, the regions imaged by the two diagnostics are displaced poloidally from each other, possibly by as much as $5 \mathrm{~cm}$. Since the field pitch is tilted at about 40 degrees to the horizontal, these imaged regions are not connected by magnetic flux. So the lack of correlation between $T_{\text {rad }} / T_{e}$ and $L_{n}$ may not be surprising in this case. For this reason, in future on NSTX we intend to combine the reflectometer and EBW emission antennas. 
Future B-X mode conversion research on NSTX will focus both on reducing $L_{n}$ to maximize $C$ and on minimizing fluctuations in $C$ by reducing the fluctuations in $L_{n} . L_{n}$ in the scrape-off can be controlled by varying the connection length, $L_{c}$, along the magnetic field lines, between the plasma limiting surfaces on either side of the EBW antenna. Assuming in this model that ambipolar diffusion across the edge field lines is balanced by particle loss to the limiters at the thermal velocity and that the diffusion in the scrape-off region is Bohm-like [18]:

$$
L_{n} \approx \sqrt{\frac{D_{B o h m}}{v_{t h, i} / L_{c}}}=\left(\left(\frac{k T_{e} L_{c}}{16 e B}\right)^{2} \frac{m_{i}}{k T_{i}}\right)^{1 / 4}
$$

Here $k$ is Boltzmann's constant, $m_{i}$ is the ion mass, $e$ is the charge on the electron, $B$ is the magnetic field and $T_{i}$ is the ion temperature. From equation 4 it can be seen that for fixed plasma parameters, $L_{n}$ varies as $L_{c}^{1 / 2}$. In the scrape off of an Ohmically-heated deuterium NSTX plasma similar to those studied here, $T_{e} \sim 2 T_{i} \sim 50 \mathrm{eV}$ and $B \sim 0.27 \mathrm{~T}$. The shortest connection length attainable in the experiment described here is probably defined by the Boron nitride tiles on either side of the EBW antenna (Fig. 1), which are separated by a distance of $45 \mathrm{~cm}$. Since the magnetic field pitch at the LCFS is 40 degrees, $L_{c} \sim 60 \mathrm{~cm}$ and equation 4 gives $L_{n} \sim 3 \mathrm{~mm}$, about half the shortest $L_{n}$ measured in the experiment described here. Since $L_{n}$ varies as $L_{c}{ }^{1 / 2}, L_{c}$ needs to be reduced to about $20 \mathrm{~cm}$ in order to make $L_{n}$ short enough to ensure $C \sim 100 \%$.

In earlier experiments on CDX-U, there was evidence that the $L_{n}$ fluctuations were reduced when insulated Boron nitride limiters were exchanged for electrically grounded Carbon limiters. Therefore, in an attempt to reduce $L_{n}$ fluctuations on NSTX, we plan to use grounded Carbon limiters with $L_{c} \sim 20 \mathrm{~cm}$ surrounding an in-vessel EBW antenna during future NSTX plasma operations, in order to demonstrate $>80 \% \mathrm{~B}-\mathrm{X}$ conversion. These measurements will test the feasibility of using a local limiter to provide high X-B conversion efficiency for future EBW heating and current drive experiments on NSTX. 


\section{ACKNOWLEDGEMENTS}

This work was supported by US Department of Energy contract no. DE-AC0276CH03073 and by a US Department of Energy research grant which is part of a program to encourage innovations in magnetic fusion energy diagnostic systems. 


\section{REFERENCES}

[1] M. Ono, S. Kaye, M. Peng, et al., Proceedings $17^{\text {th }}$ IAEA Fus. Energy Conf. (IAEA, Vienna, Austria, 1999), Vol. 3, p. 1135.

[2] J. Hosea, V. Arunasalam and R. Cano, Phys. Rev. Lett. 39, 408 (1977).

[3] J. Preinhaelter and V. Kopécky, J. Plasma Phys. 10, 1 (1973).

[4] A.K. Ram and S.D. Schultz, Phys. Plasmas 7, 4084 (2000).

[5] G. Taylor, P.C. Efthimion, B. Jones, et al., Proceedings of $14^{\text {th }}$ Topical RF Conference, AIP Conference Proceeding 595, 282 (AIP, New York, 2001).

[6] P.K. Chattopadhyay, J.K. Anderson, T.M. Biewer, et al., Phys. Plasma 9, 752 (2002).

[7] G. Taylor, P.C. Efthimion, B. Jones, et al., Phys. Plasmas 9, 167 (2002).

[8] B. Jones, P.C. Efthimion, G. Taylor, et al., "Optimized Mode Conversion of Thermally Emitted Electron Bernstein Waves (EBW) to Extraordinary Mode", Submitted to Phys. Rev. Lett. (2002).

[9] A.K. Ram, A. Bers and C.N. Lashmore-Davies, Phys. Plasmas 9, 409 (2002).

[10] J.R. Wilson, S. Bernabei, M. Carter et al., Proceedings of $13^{\text {th }}$ Topical RF Conference, AIP Conference Proceeding 485, 168 (AIP, New York, 1999).

[11] J.B. Wilgen, G.R. Hanson, T.S. Bigelow, et al., Bull. Am. Phys. Soc. 46, 259 (2001).

[12] L.L. Lao, et.al., Nucl. Fusion 25 ,1611 (1985).

[13] S.A. Sabbagh, S.M. Kaye, J. Menard, et. al., Nucl. Fusion 41, 1601 (2001).

[14] D.W. Johnson, N. Bretz, B. LeBlanc, et al., Rev. Sci. Instrum.,70, 776 (1999).

[15] G. Taylor, P.C. Efthimion, B. Jones, et al., Rev. Sci. Instrum. 72, 285 (2001).

[16] A.P. Smirnov and R.W. Harvey, Bull. Am. Phys. Soc. 40, 1837 (1995).

[17] B. Jones, PhD. Thesis, Princeton University (2002).

[18] S.J. Zweben and R.J. Taylor, Nucl. Fusion 23, 513 (1983). 


\section{TABLE CAPTION}

\section{Table I}

Measured average $T_{\text {rad }} / T_{e}$ compared to the average $C_{\max }$ and $C$ calculated by using the measured $L_{n}$ in equations (1) and (2) within the analysis time window, 0.28 to $0.32 \mathrm{~s}$. The standard deviation of the fluctuation of each quantity within the analysis time window is also indicated.

\section{FIGURE CAPTIONS}

\section{Figure 1}

Photograph showing the three circular waveguides used for X-mode reflectometry and EBW radiometry which are installed between two HHFW antenna straps and their associated Faraday shields on the midplane of NSTX. Boron nitride tiles are installed between each strap. The tiles extend $0.39 \mathrm{~cm}$ in front of the Faraday shields. Insert photograph shows a detailed enlargement of the region around the circular waveguides.

\section{Figure 2}

Schematic diagram showing a side view of the three circular waveguides shown in Fig. 1. The central waveguide is on the horizontal midplane and the other two waveguides are displaced above and below the midplane and tilted at 6 degrees to the horizontal. The upper two waveguides are used by an X-mode reflectometer that measures the electron density scrape off. The lower waveguide is used for X-mode EBW radiometry. Also shown in this figure is the outer gap between the last closed flux surface and the boron nitride tiles which was adjusted to vary the electron density scrape off length. 


\section{Figure 3}

An overlay of the time evolution of (a) the measured plasma current and (b) the outer gap distance from an EFIT equilibrium analysis for two plasmas, shot 107975 (thin line), which was programmed to have an outer gap of $6 \mathrm{~cm}$ between 0.28 and $0.32 \mathrm{~s}$ (shown shaded) and shot 107979 (thick line), which had an outer gap of less than $1 \mathrm{~cm}$ between 0.28 and $0.32 \mathrm{~s}$.

\section{Figure 4}

An overlay of (a) the $T_{e}$ and (b) $n_{e}$ profiles measured by multi-point Thomson scattering for the two shots shown in Fig 3 within the shaded time window, 0.28-0.32 s. The plotted profiles are each the result of averaging a spline fit to two, 20-point radial profiles measured at 0.293 and $0.310 \mathrm{~s}$.

\section{Figure 5}

Time evolution of the central $T_{e}$ measured by multi-point Thomson scattering and the EBW $T_{\text {rad }}$ measured at $11.6 \mathrm{GHz}$, a frequency that corresponds to emission from the magnetic axis, for the two shot shown in Figs. 3 and 4. The B-X conversion efficiency, $T_{\text {rad }} / T_{e}$, was calculated for data averaged over the analysis window between 0.28 and 0.32 s. EBW emission exhibits relatively large fluctuations that are time averaged as part of the analysis.

\section{Figure 6}

Time evolution of (a) the electron density scale length $\left(L_{n}\right)$ near the B-X conversion layer

for $11.6 \mathrm{GHz}$ EBW emission measured by $\mathrm{X}$-mode reflectometry and (b) the inferred EBW conversion efficiency, $T_{r a d} / T_{e}$, at $11.6 \mathrm{GHz}$ within the analysis time window of 0.28 to $0.32 \mathrm{~s}$ for shots 107975 and 107979 . Shot 107975 has an $L_{n}=1.94 \pm 0.46 \mathrm{~cm}$ and shot 107979 has an $L_{n}=0.72 \pm 0.28 \mathrm{~cm}$ within the analysis time window. For shot 107975 
$T_{\text {rad }} / T_{e}=10 \pm 5 \%$ and for shot 107979 has $T_{r a d} / T_{e}=38 \pm 13 \%$ within the analysis time window.

\section{Figure 7}

Results from a ray tracing analysis for $11.6 \mathrm{GHz}$ EBW rays launched from 6 degrees below the midplane. The analysis used a magnetic equilibrium at $0.301 \mathrm{~s}$ from shot 107975 and the $T_{e}$ and $n_{e}$ profiles for shot 107975 shown in Fig. 4. (a) EBW ray paths are projected (a) on a toroidal cross section through the midplane and (b) on a poloidal cross section. Rays end in the plasma when $99 \%$ of the EBW power is absorbed. The shaded region in (b) shows the region from which $11.6 \mathrm{GHz}$ EBW emission emanates.

\section{Figure 8}

Plot of the theoretical maximum B-X mode conversion efficiency, $C_{\max }$ (thin line) and the theoretical B-X mode conversion efficiency $(C)$ versus $L_{n}$ at the B-X mode conversion layer, for $11.6 \mathrm{GHz} \mathrm{EBW}$ and the edge conditions of shot 107975. Also plotted is the measured $T_{r a d} / T_{e}$ from the experiment (filled circles). Error bars indicate the fluctuation amplitude of $T_{\text {rad }}$ and $L_{n}$ within the $0.28-0.32 \mathrm{~s}$ analysis time window. 


\begin{tabular}{|c|c|c|c|c|}
\hline Shot & $\mathrm{L}_{n}(\mathrm{~cm})$ & $\mathrm{T}_{\mathrm{rad}} / \mathrm{T}_{\mathrm{e}}(\%)$ & $\mathrm{C}_{\max }(\%)$ & $\mathrm{C}(\%)$ \\
\hline 107975 & $1.95 \pm 0.45$ & $10 \% \pm 5 \%$ & $4 \% \pm 3 \%$ & $3 \% \pm 3 \%$ \\
\hline 107976 & $1.2 \pm 0.3$ & $16 \% \pm 6 \%$ & $17 \% \pm 11 \%$ & $7 \% \pm 7 \%$ \\
\hline 107977 & $0.90 \pm 0.25$ & $28 \% \pm 14 \%$ & $32 \% \pm 15 \%$ & $17 \% \pm 16 \%$ \\
\hline 107978 & $0.70 \pm 0.20$ & $33 \% \pm 10 \%$ & $50 \% \pm 21 \%$ & $39 \% \pm 25 \%$ \\
\hline 107979 & $0.70 \pm 0.25$ & $38 \% \pm 13 \%$ & $52 \% \pm 21 \%$ & $41 \% \pm 25 \%$ \\
\hline
\end{tabular}

\section{Table I}




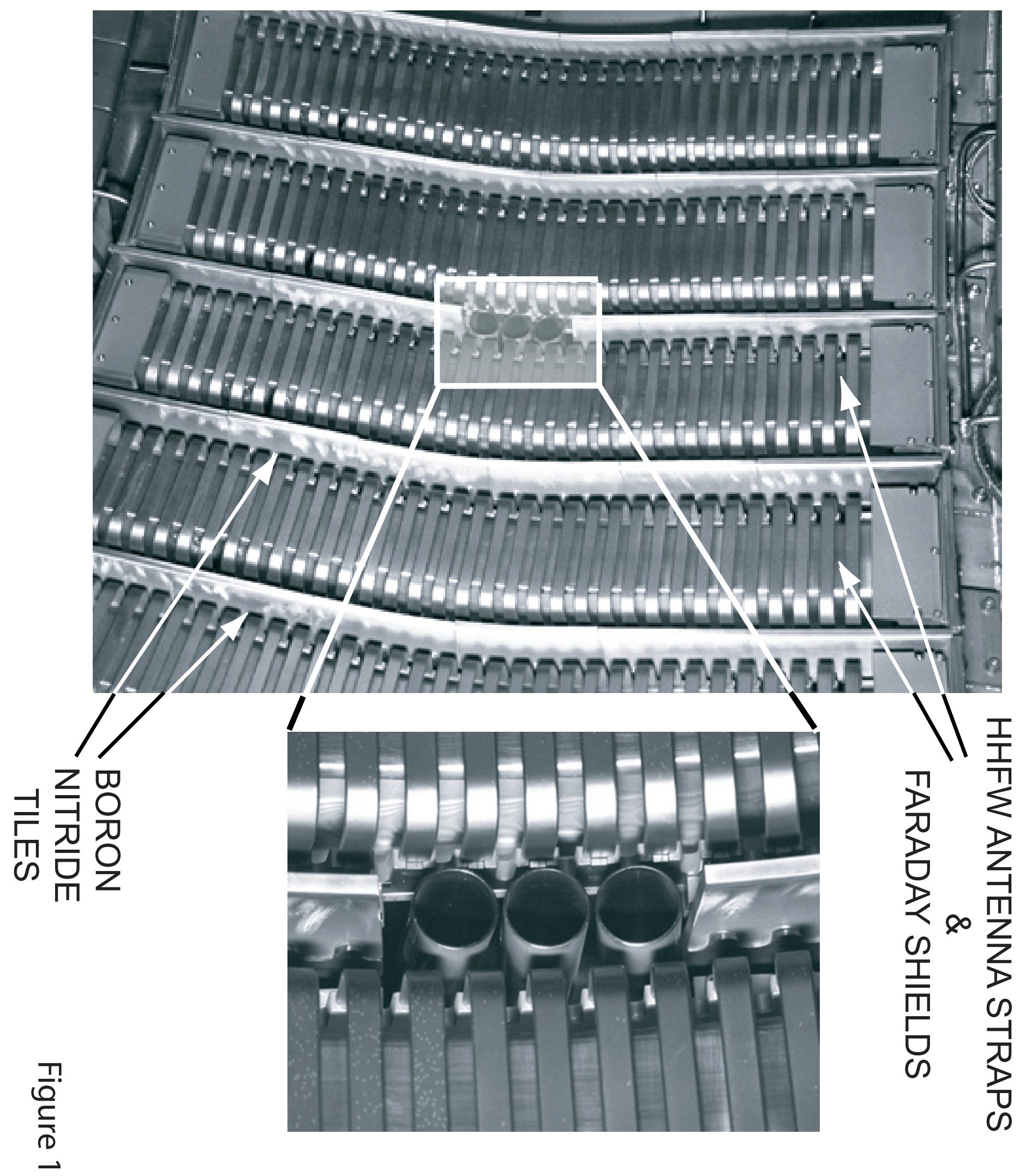




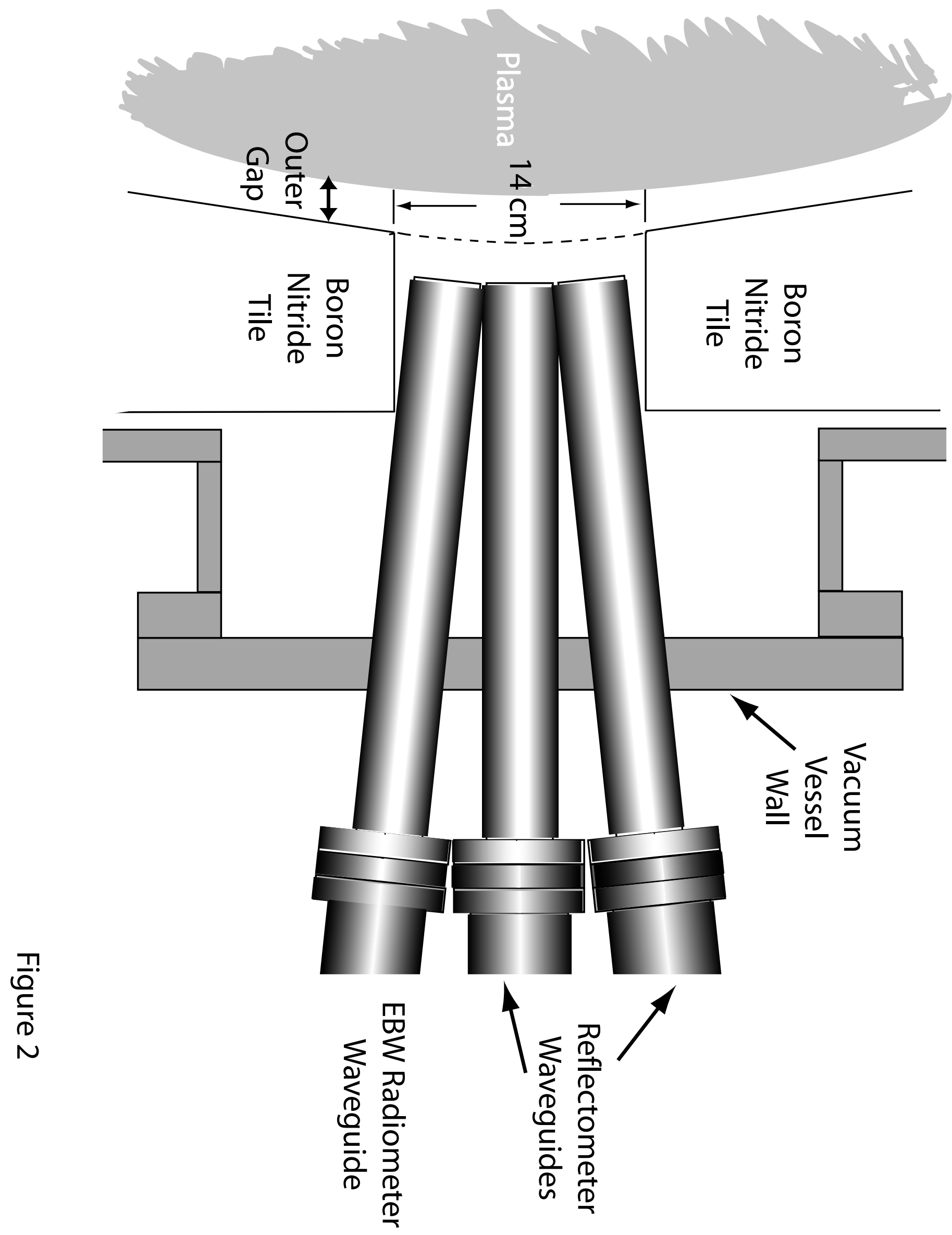



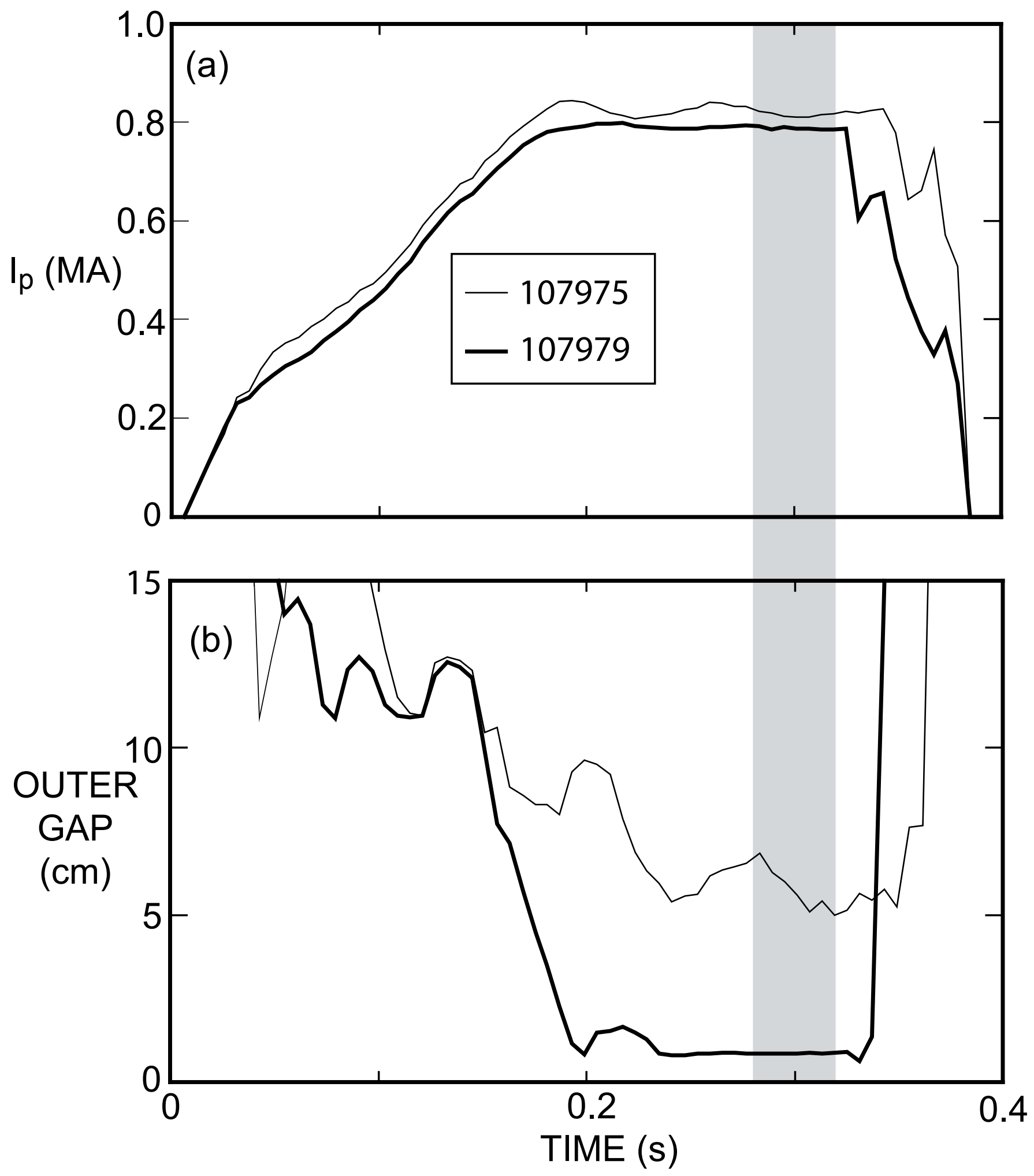

Figure 3 

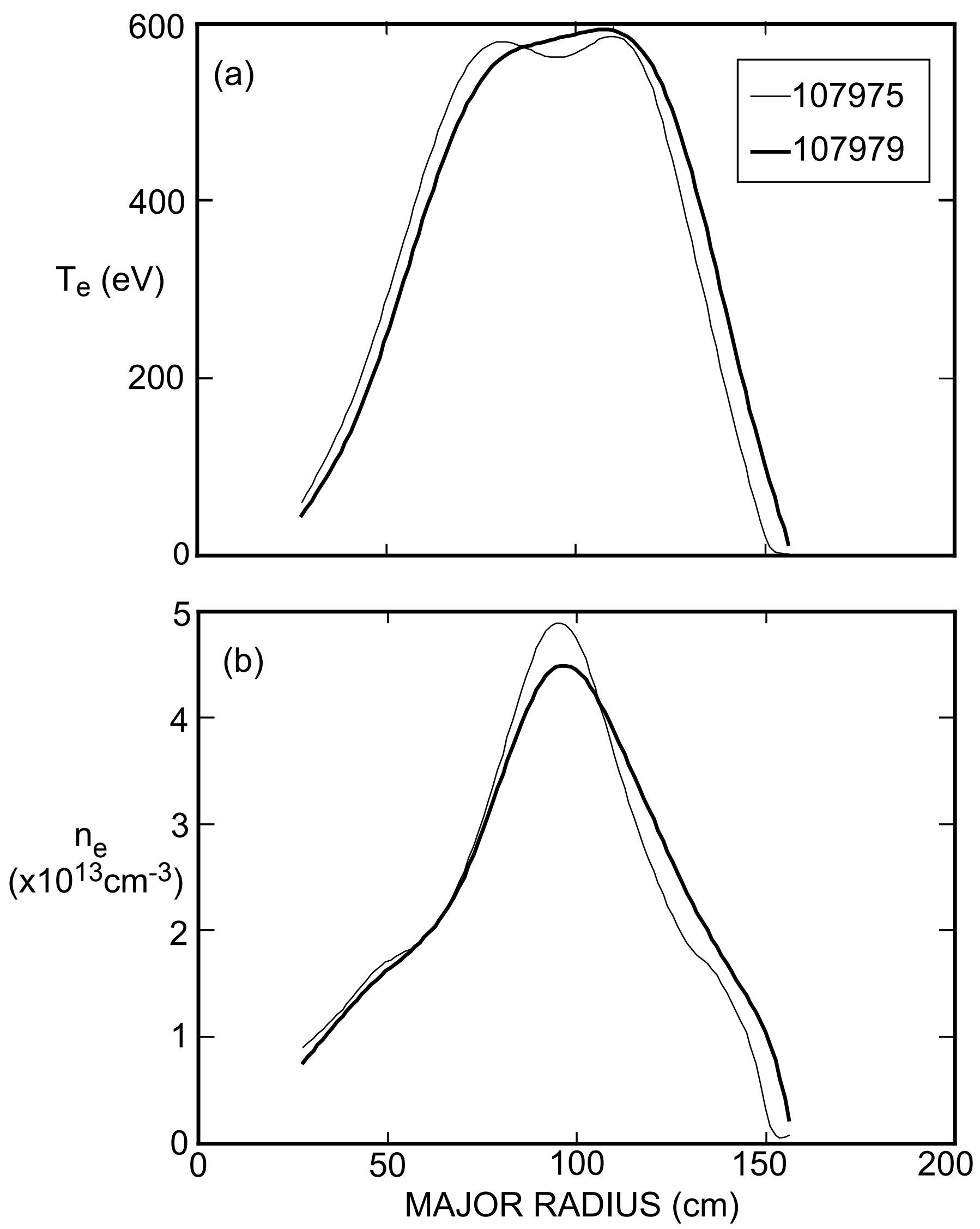

Figure 4 


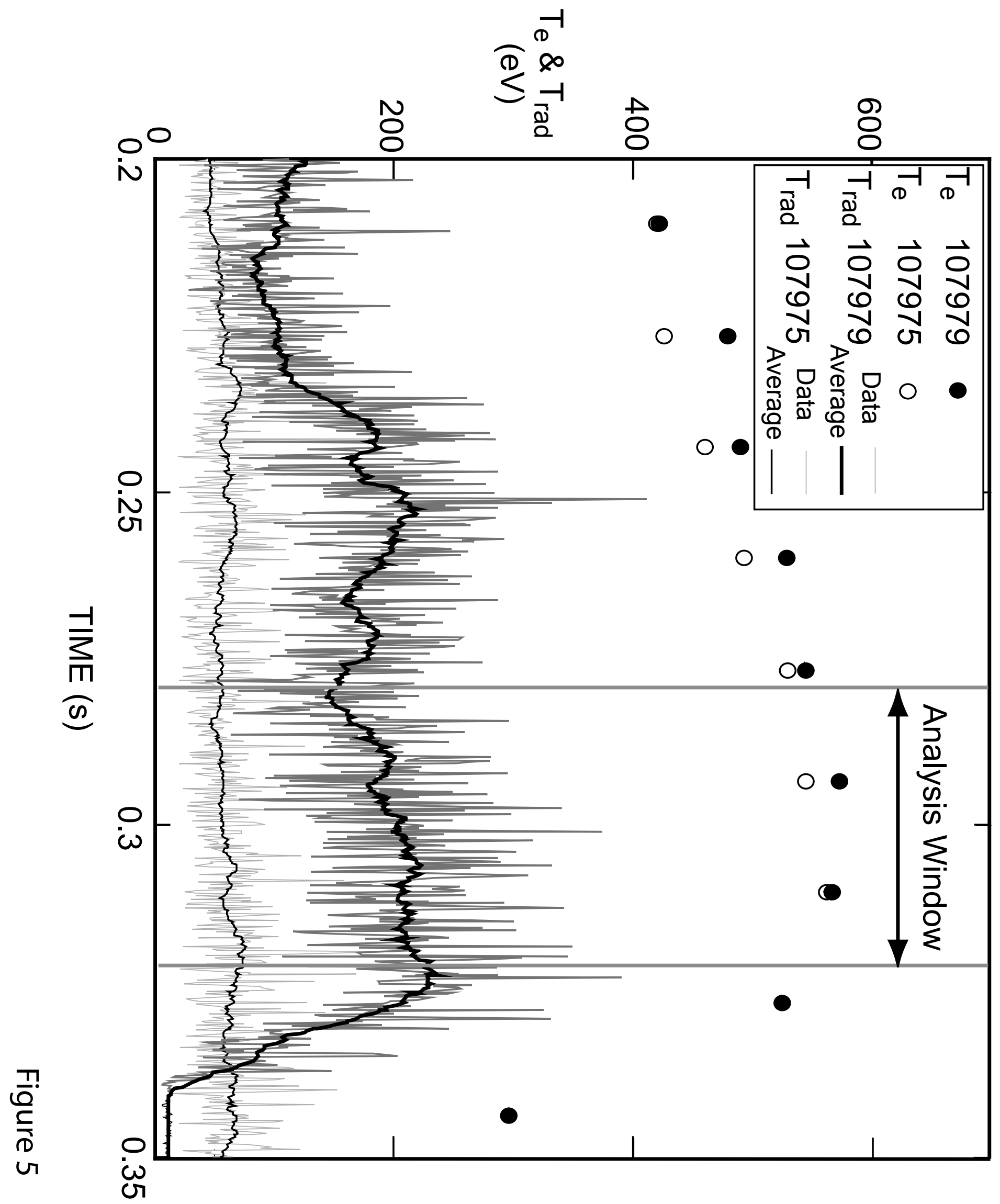




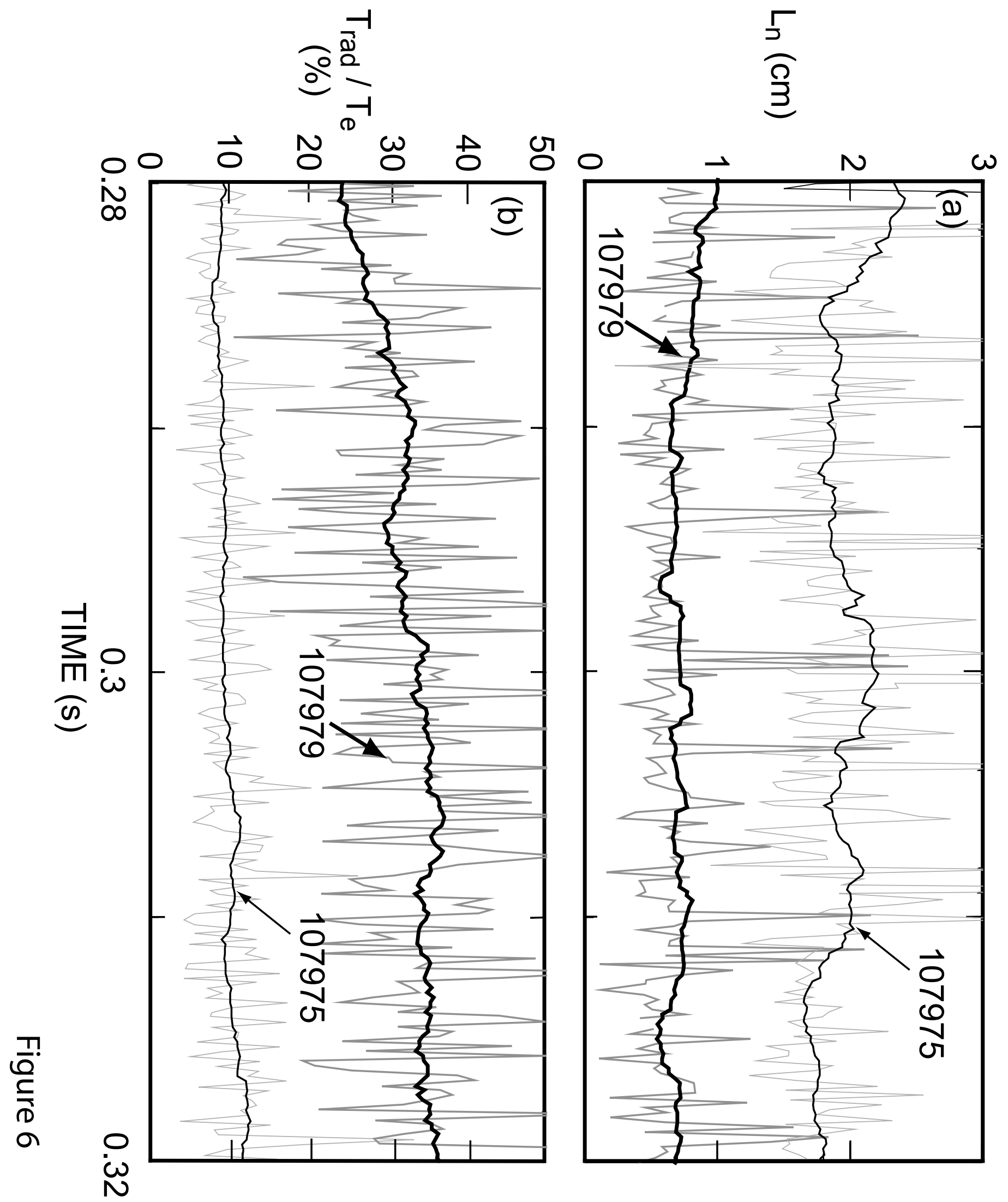



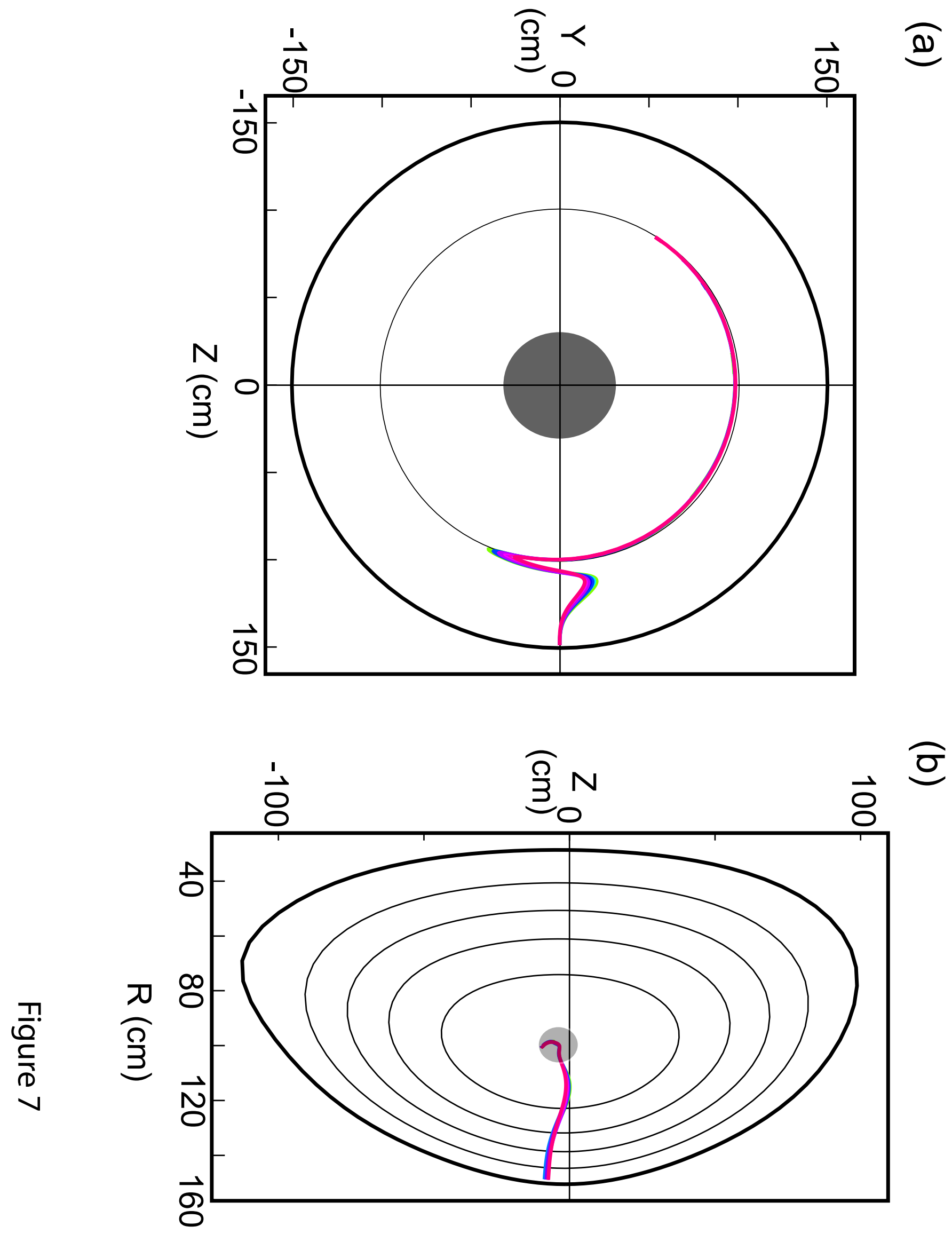


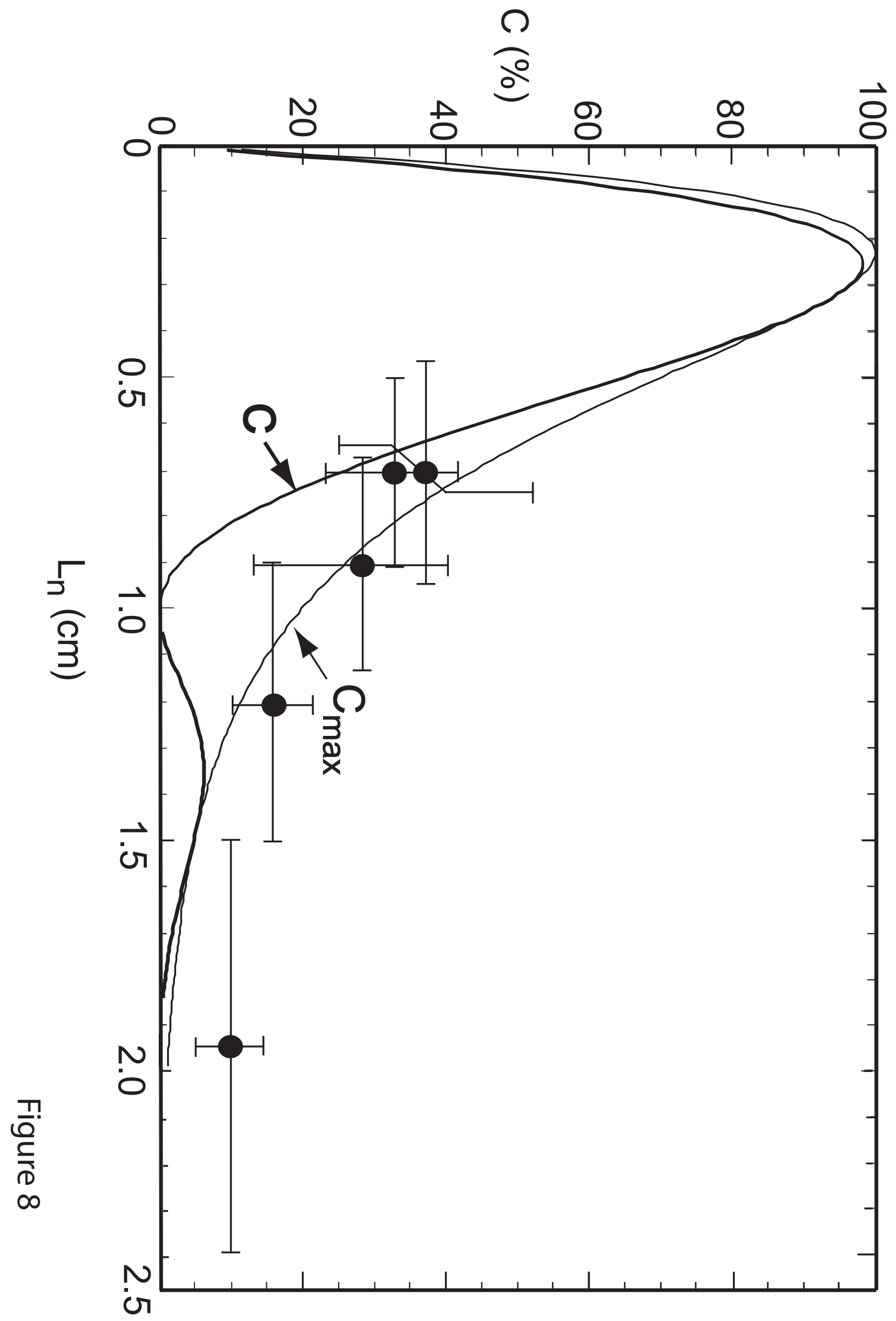




\section{External Distribution}

Plasma Research Laboratory, Australian National University, Australia

Professor I.R. Jones, Flinders University, Australia

Professor João Canalle, Instituto de Fisica DEQ/IF - UERJ, Brazil

Mr. Gerson O. Ludwig, Instituto Nacional de Pesquisas, Brazil

Dr. P.H. Sakanaka, Instituto Fisica, Brazil

The Librarian, Culham Laboratory, England

Library, R61, Rutherford Appleton Laboratory, England

Mrs. S.A. Hutchinson, JET Library, England

Professor M.N. Bussac, Ecole Polytechnique, France

Librarian, Max-Planck-Institut für Plasmaphysik, Germany

Jolan Moldvai, Reports Library, MTA KFKI-ATKI, Hungary

Dr. P. Kaw, Institute for Plasma Research, India

Ms. P.J. Pathak, Librarian, Insitute for Plasma Research, India

Ms. Clelia De Palo, Associazione EURATOM-ENEA, Italy

Dr. G. Grosso, Instituto di Fisica del Plasma, Italy

Librarian, Naka Fusion Research Establishment, JAERI, Japan

Library, Plasma Physics Laboratory, Kyoto University, Japan

Research Information Center, National Institute for Fusion Science, Japan

Dr. O. Mitarai, Kyushu Tokai University, Japan

Library, Academia Sinica, Institute of Plasma Physics, People's Republic of China

Shih-Tung Tsai, Institute of Physics, Chinese Academy of Sciences, People's Republic of China

Dr. S. Mirnov, TRINITI, Troitsk, Russian Federation, Russia

Dr. V.S. Strelkov, Kurchatov Institute, Russian Federation, Russia

Professor Peter Lukac, Katedra Fyziky Plazmy MFF UK, Mlynska dolina F-2, Komenskeho Univerzita, SK-842 15 Bratislava, Slovakia

Dr. G.S. Lee, Korea Basic Science Institute, South Korea

Mr. Dennis Bruggink, Fusion Library, University of Wisconsin, USA

Institute for Plasma Research, University of Maryland, USA

Librarian, Fusion Energy Division, Oak Ridge National Laboratory, USA

Librarian, Institute of Fusion Studies, University of Texas, USA

Librarian, Magnetic Fusion Program, Lawrence Livermore National Laboratory, USA

Library, General Atomics, USA

Plasma Physics Group, Fusion Energy Research Program, University of California at San Diego, USA

Plasma Physics Library, Columbia University, USA

Alkesh Punjabi, Center for Fusion Research and Training, Hampton University, USA

Dr. W.M. Stacey, Fusion Research Center, Georgia Institute of Technology, USA

Dr. John Willis, U.S. Department of Energy, Office of Fusion Energy Sciences, USA

Mr. Paul H. Wright, Indianapolis, Indiana, USA 
The Princeton Plasma Physics Laboratory is operated by Princeton University under contract with the U.S. Department of Energy.

\author{
Information Services \\ Princeton Plasma Physics Laboratory \\ P.O. Box 451 \\ Princeton, NJ 08543
}

Phone: 609-243-2750

Fax: 609-243-2751

e-mail: pppl_info@pppl.gov

Internet Address: http://www.pppl.gov 